\title{
BMJ Open Cuff Leak Test and Airway Obstruction in Mechanically Ventilated ICU Patients (COMIC): a pilot randomised controlled trial protocol
}

\author{
Kimberley Lewis, ${ }^{\oplus}$ Sarah Culgin, ${ }^{2}$ Roman Jaeschke, ${ }^{1,3}$ Dan Perri, ${ }^{1,4}$ \\ Corry Marchildon, ${ }^{4}$ Kelly Hassall, ${ }^{4}$ Thomas Piraino, ${ }^{5}$ Lehana Thabane, ${ }^{2,3}$ \\ Yousef Almubarak, ${ }^{6}$ Mohammed S Alshahrani, ${ }^{\circ}$ Bram Rochwerg, ${ }^{1,3}$ \\ Bandar Baw, ${ }^{1,8}$ Wojciech Szczeklik, ${ }^{9}$ Tim Karachi, ${ }^{1}$ Waleed Alhazzani, ${ }^{1,3,4}$ For the \\ GUIDE Group
}

To cite: Lewis $\mathrm{K}$, Culgin $\mathrm{S}$, Jaeschke R, et al. Cuff Leak Test and Airway Obstruction in Mechanically Ventilated ICU Patients (COMIC): a pilot randomised controlled trial protocol. BMJ Open 2019:9:e029394. doi:10.1136/ bmjopen-2019-029394

- Prepublication history for this paper is available online. To view these files, please visit the journal online (http://dx.doi. org/10.1136/bmjopen-2019029394).

Received 24 January 2019 Revised 17 June 2019 Accepted 25 June 2019

\section{Check for updates}

(c) Author(s) (or their employer(s)) 2019. Re-use permitted under CC BY-NC. No commercial re-use. See rights and permissions. Published by BMJ.

For numbered affiliations see end of article.

\section{Correspondence to}

Dr Waleed Alhazzani; waleed.al-hazzani@medportal. ca

\section{ABSTRACT}

Introduction Endotracheal intubation and invasive mechanical ventilation are lifesaving interventions that are commonly performed in the intensive care unit (ICU). Laryngeal oedema is a known complication of intubation that may cause airway obstruction in a patient on extubation. To date, the only test available to predict this complication is the cuff leak test (CLT); however, its diagnostic accuracy and utility remains uncertain. Herein, we report the protocol for the CuffLeak and AirwayObstruction in MechanicallyVentilated ICU Patients (COMIC) pilottrial.

Methods and analysis This will be a multicentred, pragmatic, pilot randomised controlled trial (RCT). We will enrol 100 mechanically ventilated patients in the ICU who are deemed ready for extubation. We will exclude patients at a high risk of laryngeal oedema. All enrolled patients will have a CLT done before extubation. In the intervention arm, the results of the CLT will be communicated to the bedside physician, and decision to extubate will be left to the treating team. In the control arm, respiratory therapist will not communicate the results of the CLT to the treating physician, and the patient will be extubated regardless of the CLT result. Randomisation will be done in a 1:1 allocation ratio, stratified by size of the endotracheal tube and duration of invasive mechanical ventilation. Although we will examine all clinical outcomes relevant for the future COMIC RCT, the primary outcomes of the COMIC pilottrial will be feasibility outcomes including: consent rate, recruitment rate and protocol adherence. Clinical outcomes include postextubation stridor, reintubation, emergency surgical airway, ICU mortality, in hospital mortality, duration of mechanical ventilation and ICU length of stay in days.

Ethics and dissemination The Hamilton Integrated Research Ethics Board, Imam Abdulrahman Bin Faisal University Institutional Review Board and Bioethical Commission of the Jagiellonian University approved this study. The trial results will be disseminated via publication in peer-reviewed journals.

Trial registration number NCT03372707.

\section{Strengths and limitations of this study}

- This is the first pilot randomised trial to assess feasibility and impact of performing the cuff leak test (CLT) before extubation in mechanically ventilated patients at average risk of laryngeal oedema.

- Information from this study will facilitate the conduct of a larger trial powered to determine the impact of the CLT on patient-important outcomes.

- The multicentred, international design will support external validity and implementation of the results

- As the most accurate way to define a failed or passed CLT is unknown (ie, quantitatively vs qualitatively), we are defining a failed CLT as inability to auscultate air movement around the endotracheal tube with the cuff deflated.

\section{INTRODUCTION}

Endotracheal intubation and invasive mechanical ventilation are lifesaving interventions; however, it can be associated with serious complications. Laryngeal oedema (LO) occurs in 4\%-55\% of patients postextubation. ${ }^{1-5}$ LO is caused by marked polymorphonuclear infiltration to the traumatised upper airway postintubation. ${ }^{6}$ The incidence of LO increases as the duration of intubation accrues, but it can occur as early as the first 24 hours of intubation. ${ }^{5}$ LO can cause airway narrowing and increased airflow velocity, if the narrowing exceeds $50 \%$ of the lumen diameter, leading to stridor and respiratory distress postextubation. ${ }^{7}$ As a result, 3.5\% (range $0 \%-10.5 \%$ ) of patients with LO will fail extubation and subsequently require reintubation. ${ }^{5}$ The morbidity and mortality associated with reintubation are well described. ${ }^{8-13}$

Identifying patients with LO can be challenging. The presence of the endotracheal 
tube (ETT) prevents direct visualisation of the upper airway before extubation; therefore, clinicians cannot accurately predict airway obstruction before it occurs. A cuff leak test (CLT) was first described in 1988 as a surrogate and a screening test for airway oedema before extubation. ${ }^{14}$ This test involves deflating the balloon cuff on an ETT and observing if the patient can breathe around it. If air can pass around the ETT, it suggests that the airway is patent. ${ }^{14} \mathrm{~A}$ small leak or complete absence of one would suggest an airway obstruction or narrowing.

There are conflicting results on the clinical utility and diagnostic accuracy of a CLT. Two meta-analyses of observational studies examined the diagnostic accuracy of a CLT. ${ }^{515}$ One reports that a failed CLT is not sensitive but is specific for predicting risk of $\mathrm{LO}$ and reintubation. ${ }^{15}$ The second meta-analysis found that a failed CLT was associated with LO, particularly in patients with $>5$ days' duration of intubation; however, the odds of reintubationwas not increased..$^{5}$

Despite the lack of high-quality studies, an absent cuff leak usually results in delayed extubation and exposure to medications aimed at treating airway oedema. A recent meta-analysis of 11 randomised controlled trials (RCTs) enrolling 2472 patients found that systemic corticosteroids reduces the risk of postextubation airway events (relative risk (RR) $0.43,95 \%$ CI 0.29 to $0.66, p=0.002) .{ }^{16}$ A subgroup analysis showed that the high-risk subgroup benefited the most (RR $0.34,95 \%$ CI 0.24 to 0.48 , $\mathrm{p}=0.99$ ). Empirical use of corticosteroids for all patients is not justified (RR $0.62,95 \%$ CI 0.24 to $1.61, \mathrm{p}<0.001$ ). A false positive CLT can unnecessarily delay extubation, increasing intensive care unit (ICU) stay and associated risks of invasive mechanical ventilation. However, if a CLT is not performed, or if in case of a false negative test, some patients may fail the extubation exposing them to the morbidity associated with reintubation.

Recent clinical practice guidelines reflect this uncertainty. The American Thoracic Society guidelines on liberation of mechanical ventilation issued a weak recommendation (very low quality evidence) for performing CLT in mechanically ventilated adults who are at high risk for postextubation stridor (such as airway trauma) and those with a potential for increased risk of LO (eg, intubated for 7 days or more).${ }^{17}$ Given this persistent uncertainty, we believe a properly powered RCT is necessary to investigate the clinical utility of the CLT and its impact on patient outcomes. Our aim is to report the protocol for the CuffLeak and AirwayObstruction in MechanicallyVentilated ICU Patients (COMIC) pilottrial to determine the feasibility of undertaking a large RCT addressing this research question. Given the clinical equipoise, we hypothesise that this will be a feasible trial, and the results will inform the larger COMIC RCT.

\section{METHODSANDANALYSIS}

We registered this trial in ClinicalTrials.gov.
Table 1 Exclusion criteria

\section{Exclusion criteria Definitions}

$\begin{array}{ll}\text { 1. Palliative care } & \text { Decision to withdraw life support or no } \\ \text { plan or plan of care } & \text { plan for reintubation. } \\ \text { does not include } & \\ \text { reintubation } & \end{array}$

Known

pregnancy

Current pregnancy or up to and including 7 days postpartum.

3. High risk patient
for LO

for LO

Burn patients, smoke inhalation injuries, blunt or penetrating trauma of the neck and airway, recent head and neck surgeries, self-extubation event and patients admitted with airway oedema. ${ }^{25-27}$

4. Difficult or Direct laryngoscopy Cormack-Lehane traumatic intubation Grade 4 (regardless of the number of intubation attempts); three or more attempts at intubation regardless of the grade; an intubating supraglottic device, Bougie or bronchoscopy required previously to intubate; or unable to bag mask ventilate.

$\begin{array}{ll}\text { 5. Known } & \text { Vocal cord paralysis, tracheolaryngeal } \\ \text { pre-existing } & \text { neoplasm, tracehomalasia, } \\ \text { tracheolaryngeal } & \text { tracheolaryngeal stenosis or previous } \\ \text { abnormalities } & \text { head and neck surgeries. }\end{array}$

6. Mechanical ventilation via a tracheostomy

7. Patients who failed extubation attempt within the current ICU admission.

8. History of postextubation airway obstruction.

9. The ICU physician declined enrolling the patient.

10. Patient had a failed CLT in the previous 24 hours.

CLT, cuff leak test; ICU, intensive care unit; LO, laryngeal oedema.

\section{Design}

The COMIC pilot trial will be a multicentre, randomised, concealed, parallel-group, pragmatic pilot trial. Three centres from North America, Europe and the Middle East will participate in the COMIC pilot trial. These academic tertiary care ICUs are located in Hamilton, Canada; Krakow, Poland; and Dammam, Saudi Arabia.

\section{Population}

Eligible patients will be mechanically ventilated adults ( $>18$ years) in the ICU, and an order to extubate has been provided by the treating physician. We list the exclusion criteria in table 1 .

\section{Eligible non-randomised patients}

We will record all patients who were eligible but not randomised for any of the following reasons: (1) thepatient or substitute decision maker (SDM) declined consent; (2) there are no family members or SDM for the patient; (3) the ICU physician declined enrolling the patient; and (4) any other reason. 


\section{Randomisation and allocation concealment}

The study researchcoordinator (RC) will use the REDCap randomisation module to randomise eligible patients in a 1:1 allocation using undisclosed variable block sizes. ${ }^{18}$ We will stratify randomisation by: (A) ETT size into two strata (external diameter equivalent to that of $<8 \mathrm{~mm}$ and $\geq 8 \mathrm{~mm}$ ETTs), (B) duration of mechanical ventilation before randomisation into two strata ( $>7$ days and $\leq 7$ days) and (C) by study site.

\section{Intervention}

A dedicated unblinded study respiratorytherapist (RT) or a trained researchpersonnel (referred to as an RT from here on) will perform the CLT on all enrolled patients. The patients will first be switched to volume assist-control (V-AC) with a set respiratory rate of 10 breaths/minute (to allow patient assist), a constant flow of $60 \mathrm{~L} / \mathrm{min}$ and tidal volume set to match the average tidal volume currently being delivered during supportive ventilation. The RT will document one representative inhaled tidal volume and three exhaled volumes after switching to V-AC mode. The cuff of the ETT will then be deflated with a $10 \mathrm{~mL}$ syringe. Once the patient has accommodated to the deflated cuff, we will record the inspiratory and expiratory volumes of three consecutive breaths. The cuff leak test is performed by: (A) auscultation with a stethoscope to identify audible air leak around the ETT, (B) determining the difference between the average exhaled volume prior to cuff deflation and the average exhaled volume after cuff deflation, $(\mathrm{C})$ measure the difference between the average inhaled and exhaled volumes after cuff deflation and (D) calculate the percent change in expiratory volume with the cuff deflated.

\section{(i.e.percent change}

$=\frac{\text { Expiratory tidal voluume }}{\text { axallon inflated }- \text { Expiratory tidal volume }_{\text {balloon deflated }}}$ )

We define a 'failed CLT' as the RT being unable to identify air leak during auscultation. Patients randomised to the intervention arm will have the results of the CLT (whether failed or passed) communicated to the treating physician; the treating physician will decide whether to proceed with extubation based on the CLT results. It is at the discretion of the treating physician to provide corticosteroids at any dose or frequency and/or delay extubation by 24 hours should the patient fail the CLT. ${ }^{19}$ Those randomised to the control arm will not have the results of the CLT (whether failed or passed) communicated to the treating team and will be extubated regardless of the results (figure 1).

\section{Blinding}

Although the intervention arm of the study is unblinded (ie, results of CLT is known), patients, physicians, RCs, study investigators, adjudicators and data analysts will not be aware of the results of CLT in the control group. The unblinded RT will document the CLT results on the CLT paper case report form (CRF). The RT will enter the CLT

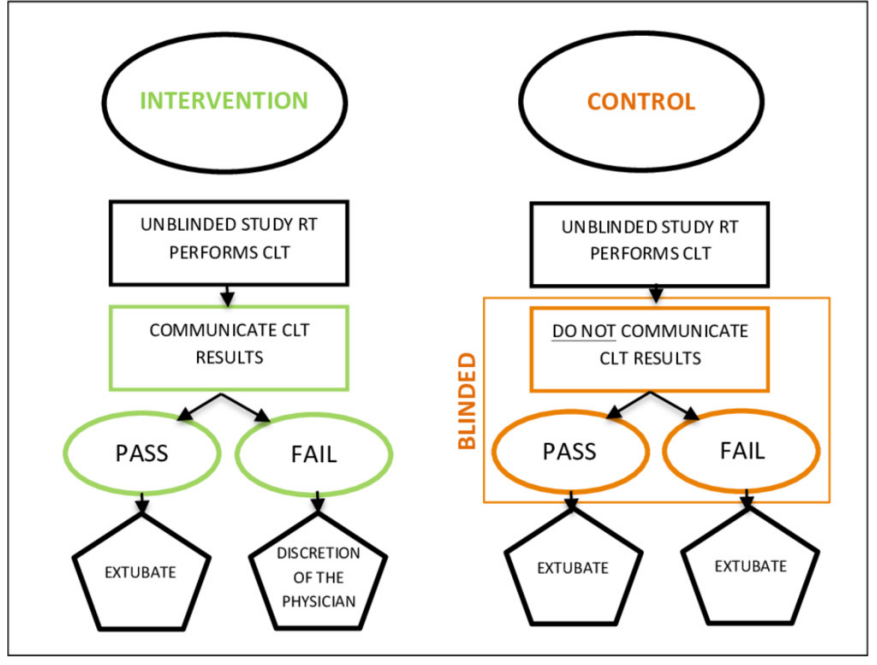

Figure 1 Flow chart demonstrating the two groups that a patient may be randomised into and course of action for each group depending on if the patient fails or passes their cuff leak test. CLT, cuff leak test; RT, respiratory therapist.

results data into the electronic CRF (eCRF) in the trial database on REDCap. The eCRF for the CLT will only be accessible and visible to the RT. For both the intervention and control arms, the paper CRF will be stored in the patient binder. For patients randomised to the control $\mathrm{arm}$, the RT will seal the paper CRF in an envelope before storing in the patient binder. In case of emergency, and if knowing the results of the CLT will change the management of the patient (eg, administering corticosteroids to a patient who developed respiratory failure after learning the result of a failed CLT), the treating physician will have the ability to request the CLT results for a control arm patient. In such an event, the sealed paper CRF can be opened to reveal the CLT results to the treating physician and patient care team, and the methods centre must be notified of this protocol violation.

\section{Patients and public involvement}

Patients and the public were not involved in the design or the conduct of this study. Once the study is published, we plan to involve patient representatives in the Data Safety Monitoring Board in the larger COMIC Trial.

\section{OUTCOMES}

\section{Feasibility outcomes}

Although we will report all clinical outcomes relevant for the future COMIC RCT, the primary outcomes of the COMIC pilottrial will be feasibility, including:

\section{Consent rate}

We define a successful consent rate as $70 \%$ of SDMs or patients approached to consent, choosing to take part in the trial. We will calculate this as the overall proportion of SDMs or patients consenting out of those approached (with 95\% CI). As this is a pilottrial, the steeringcommittee will review the consent rate weekly, and if applicable, 
discuss barriers to informed consent and use factors associated with improved consent rate. ${ }^{20}$

\section{Recruitment rate}

We define a successful recruitment rate as achieving enrolment of 100 patients, conventionally expressed as four patients per centre per month over the duration of the trial. While the pilot trial is ongoing, steeringcommittee will review recruitment weekly and the screening records monthly. If applicable, we will address barriers to enrolment to maximise recruitment. The recruitment metric will be measured and interpreted at the end of the pilot trial by calculating the mean number of recruited patients per active screening month.

\section{Protocol adherence}

We define a successful adherence as $\geq 80 \%$. We will calculate the adherence as the proportion of patients that were assigned to the control arm being extubated immediately after the CLT. As this pilot trial is ongoing, we will review adherence monthly and investigate the reasons for compliance failure. We will investigate all reasons for failure to extubate immediately after randomisation in the control arm and report them as a protocol violation. The RC will review the RT's charting and the medication profile to determine actual compliance. $\mathrm{RC}$ will record all reasons for non-compliance for both groups using distinguishing clinical reasons (eg, palliation, death, consent withdrawal and errors).

\section{Clinical outcomes}

The clinical outcomes are:

(1) Postextubation stridor: defined as an audible highpitched inspiratory noise caused by turbulent airflow through the narrowed airway that is detectable with or without a stethoscope within 48 hours of extubation; (2) clinically significant postextubation stridor: defined as stridor (see definition above) that requires medical intervention such as the administration of systemic steroids, racemic epinephrine, Heli-ox or reintubation; (3) reintubation: defined as reintubation within 72 hours of original extubation while in the ICU. Reasons for reintubation will be recorded; (4) emergency surgical airway: defined as performing urgent tracheostomy or cricothyrotomy for a life-threatening airway obstruction; (5) in ICU mortality truncated at 30 days; (6) in-hospital mortality truncated at 30 days; (7) duration of mechanical ventilation: defined as time on the ventilator after randomisation in days; and (8) ICU length of stay in days.

\section{Data collection and follow-up}

The RC will screen all patients in the ICU during weekdays to avoid incurring additional weekend on-call costs. $\mathrm{RC}$ will collect information including the patient baseline data (eg, demographics, illness severity, advanced life support including duration of mechanical ventilation, daily data (eg, CLT results, postextubation stridor, rate of reintubation and steroids administered) and source documentation that will help with the adjudication of outcomes. The RC will review patient's charts daily for up to 72 hours postextubation for the trial data including: stridor, reintubation and emergency surgical airway. Once patients are discharged from the ICU, they will no longer be followed daily, but we will record the patients' vital status at 30 days if the patient was not discharged from the hospital. Mortality, duration of ventilation and ICU stay outcomes will be censored at 30 days.

\section{Duration of the COMIC pilot trial}

We began enrolling patients on 5 July 2018. We expect that 12 months will be required to recruit 100 patients, with a trial end date of approximately June 2019. We will need a subsequent 3 months to validate the data, adjudicate the outcomes, analyse, interpret and present results. The total duration of the pilottrial may take up to 15 months to complete.

\section{Sample size and justification}

We plan to enrol 100 patients for the pilot trial to ensure feasibility criteria will be appropriately examined. ${ }^{21}{ }^{22}$

\section{Analysis of the COMIC pilot trial}

The analysis and reporting of this pilot trial will follow the Consolidated Standards of Reporting Trials extension to pilot trials. ${ }^{23}$ We will use descriptive statistics to analyse the baseline characteristics and report them as a count (per cent) for categorical variables, and mean (SD) or median (first quartile, third quartile) for continuous variables, depending on the distribution. We will base the analysis of feasibility outcomes on descriptive statistics reported as percentages with $95 \%$ CI. Calculation of consent and recruitment feasibility outcomes for the COMIC pilottrial will not require analysis by group; however, compliance rates will have to be assessed for each group. Therefore, we will analyse clinical outcomes as means or proportions in each arm. In addition, given the small sample size and short duration, we will not conduct any subgroup or interim analyses.

The analysis of clinical outcomes will follow intentionto-treat approach. These analyses will be exploratory. The proportion of patients in the two groups with the primary and secondary clinical outcomes will be analysed using the Mantel-Haenszel $\chi^{2}$ test of Fisher's exact test. A t-test will be used for continuous outcomes, and a statistical significance will be set at alpha $=0.05$. These analyses will be exploratory, and the results will be reported as estimates of effect with 95\% CIs. We will develop a full statistical analysis plan adherent to the intention-to-treat principle. All analyses will be performed using SAS V.9.2.

\section{ETHICSANDDISSEMINATION \\ Ethics}

Centres will follow the consent models outlined below, depending on the local ethics approval.

Mixed consent model (a priori and deferred consent)

As most patients will be incapable at the time of enrolment, the SDM will provide consent a priori whenever 
possible. We will follow the two-phase, 13-step informed consent process that we have used in prior international trials. ${ }^{20}$

If we cannot locate the SDM for a priori consent, then we will enrol patients using deferred consent until we can contact the SDM as permitted by local Research ethics board (REBs). The consent encounter will occur as soon as possible. If the SDM then declines further trial proceedings data collected will be used up to that point unless the SDM requests otherwise. For the patient who has no identifiable family member, or power of attorney to provide consent, we propose to continue study protocols while we attempt to locate an SDM and/or the patient recovers. In the event that such a patient subsequently recovers to the extent thatan informed consent can be provided, we will ask the patient for their consent.

Having a mixed consent model is crucial to ensure the proper conduct of the COMIC trial. To begin with, extubating patients is a daily routine in the ICU and should not be delayed unless there is a valid reason to do so. Therefore, having either a waved or deferred consent model is necessary to make this study feasible, and we have successfully used deferred consent model in a recent RCT. $^{24}$

Moreover, although CLT is routinely performed in some institutions before extubation, there is no clear evidence that this practice benefit patients. ${ }^{5}$ The ethical principle of clinical equipoise underlies all medical research and obligates researchers to provide standard treatment unless there is uncertainty about the relative effectiveness of the standard and experimental treatments. Recent clinical practice guidelines for using CLT reflect this uncertainty. ${ }^{17}$ In this case, it can be argued the relative benefits and risks of the CLT, as compared with standard therapy, are unknown, or thought to be equivalent or better.

\section{Waived consent model}

Certain participating centres may receive approval (under the justification that clinical equipoise exists and the test itself is harmless) to waive consent. This is conditional on REB approval at those participating centres.

We will keep all personal information in a locked room. No personal information is available on REDCap.

\section{Dissemination and protocol amendments}

We will submit the primary RCT results for publication to a peer-reviewed journal. If the protocol needs amendment, investigators are required to inform the institutional REB (as well aspatients) and receive approval.

\section{DISCUSSION}

Extubation can be a precarious procedure for patients admitted to the ICU. Therefore, critical care physicians undertake the utmost cautions before extubating patients. The CLT is the most commonly used test to detect LO in mechanically ventilated patients. However, the diagnostic accuracy and impact on clinical outcomes in average risk patients is unclear. Recent guidelines issued a weak recommendation to perform CLT before extubating high-risk patients. ${ }^{17}$ Patients who fail a CLT are often treated with high-dose systemic corticosteroids and extubation may be delayed; both outcomes are likely not desirable by most patients.

To date, no RCT has been done examining this important question. Observational studies have showed that in a subset of patients a CLT may help identify patients at higher risk of airway obstruction. We therefore have described the protocol for a pilot RCT to determine the feasibility of a large trial to examine the utility of CLT. Physician and public acceptance of performing such a protocol is uncertain, and it is integral we examine the recruitment, consent and adherence rates before we pursue a large-scale RCT.

In conclusion, this protocol describes the design and methodology of the COMIC pilottrial. We believe the results will help inform the design and the conduct of a large RCT examining the effect of bedside CLT on postextubation events in average risk mechanically ventilated patients.

\section{Author affiliations}

${ }^{1}$ Department of Medicine, McMaster University, Hamilton, Ontario, Canada ${ }^{2}$ The Research Institute of St. Joseph's Healthcare Hamilton, St Joseph's Healthcare, Hamilton, Ontario, Canada

${ }^{3}$ Department of Health Research Methods, Evidence, and Impact, McMaster University, Hamilton, Ontario, Canada

${ }^{4}$ St. Joseph's Healthcare, Hamilton, Ontario, Canada

${ }^{5}$ Department of Critical Care, St Michael's Hospital, Toronto, Ontario, Canada ${ }^{6}$ Department of Critical Care, Imam Abdulrahman Ben Faisal University, Dammam, Saudi Arabia

${ }^{7}$ Department of Emergency and Critical Care, Imam Abdulrahman Ben Faisal University, Dammam, Saudi Arabia

${ }^{8}$ Department of Medicine, Division of Emergency Medicine, McMaster University, Hamilton, Ontario, Canada

${ }^{9}$ Department of Intensive Care and Perioperative Medicine, Jagiellonian University Medical College, Krakow, Poland

Acknowledgements We would like to acknowledge the ICU Respiratory Therapists and Registered Nurses at St Joseph's Healthcare, Hamilton, Ontario, that helped to plan and implement the protocol. We would like to especially recognise France Clarke for her assistance.

Contributors WA and KL conceived the study. All authors contributed to protocol development. KL and WA drafted the protocol. KL and WA are grant holders. LT provided statistical expertise. All authors contributed to refinement of the study protocol and approved the final manuscript.

Funding Funding was provided from the McMaster Internal Medicine Dr Paul O'Byrne Grant, the McMaster Critical Care Fellowship programme and Guidelines in Intensive Care, Development and Evaluation Group at St. Joseph's Healthcare, Hamilton, Canada.

Competing interests None declared.

Patient consent for publication Not required.

Ethics approval The Hamilton Integrated Research Ethics Board, Imam Abdulrahman Bin Faisal University Institutional Review Board, and Bioethical Commission of the Jagiellonian University approved of the protocol.

Provenance and peer review Not commissioned; externally peer reviewed.

Open access This is an open access article distributed in accordance with the Creative Commons Attribution Non Commercial (CC BY-NC 4.0) license, which permits others to distribute, remix, adapt, build upon this work non-commercially, 
and license their derivative works on different terms, provided the original work is properly cited, appropriate credit is given, any changes made indicated, and the use is non-commercial. See: http://creativecommons.org/licenses/by-nc/4.0/.

\section{REFERENCES}

1 Maury E, Guglielminotti J, Alzieu M, et al. How to identify patients with no risk for postextubation stridor? J Crit Care 2004;19:23-8.

2 Tadié JM, Behm E, Lecuyer L, et al. Post-intubation laryngeal injuries and extubation failure: a fiberoptic endoscopic study. Intensive Care Med 2010;36:991-8.

3 Sutherasan $\mathrm{Y}$, Theerawit $\mathrm{P}$, Hongphanut $\mathrm{T}$, et al. Predicting laryngeal edema in intubated patients by portable intensive care unit ultrasound. J Crit Care 2013;28:675-80.

4 François B, Bellissant E, Gissot V, et al. 12-h pretreatment with methylprednisolone versus placebo for prevention ofpostextubation laryngeal oedema: a randomised double-blind trial. Lancet 2007;369:1083-9.

5 Zhou T, Zhang HP, Chen WW, et al. Cuff-leak test for predicting postextubation airway complications: a systematic review. J Evid Based Med 2011;4:242-54.

6 Way WL, Sooy FA. LXV Histologic changes produced by endotracheal intubation. Annals of Otology, Rhinology \& Laryngology 1965;74:799-812.

7 Mackle T, Meaney J, Timon C. Tracheoesophageal compression associated with substernal goitre. Correlation of symptoms with cross-sectional imaging findings. J LaryngolOtol 2007;121:358-61.

8 Epstein SK, Ciubotaru RL. Independent effects of etiology of failure and time to reintubation on outcome for patients failing extubation. Am J Respir Crit Care Med 1998;158:489-93.

9 Demling $\mathrm{RH}$, Read T, Lind LJ, et al. Incidence and morbidity of extubation failure in surgical intensive care patients. Crit Care Med 1988;16:573-7.

10 Esteban A, Alía I, Gordo F, et al. Extubation outcome after spontaneous breathing trials with T-Tube or pressure support ventilation. Am J Respir Crit Care Med 1997;156:459-65.

11 Frutos-Vivar F, Esteban A, Apezteguia C, et al. Outcome of reintubated patients after scheduled extubation. J Crit Care 2011;26:502-9.

12 Torres A, Gatell JM, Aznar E, et al. Re-intubation increases the risk of nosocomial pneumonia in patients needing mechanical ventilation. Am J Respir Crit Care Med 1995;152:137-41.
13 Seymour CW, Martinez A, Christie JD, et al. The outcome of extubation failure in a community hospital intensive care unit: a cohort study. Crit Care 2004;8:R322-7.

14 Potgieter PD, Hammond JM. "Cuff" test for safe extubation following laryngeal edema. Crit Care Med 1988;16:818.

15 Ochoa ME, Marín MC, Frutos-Vivar F, et al. Cuff-leak test for the diagnosis of upper airway obstruction in adults: a systematic review and meta-analysis. Intensive Care Med 2009;35:1171-9.

16 Kuriyama A, Umakoshi N, Sun R. Prophylactic corticosteroids for prevention ofpostextubation stridor and reintubation in adults: a systematic review and meta-analysis. Chest 2017;151:1002-10.

17 Girard TD, Alhazzani W, Kress JP, et al. An Official American Thoracic Society/American College of Chest Physicians Clinical Practice Guideline: liberation from mechanical ventilation in critically ill adults. rehabilitation protocols, ventilator liberation protocols, and cuff leak tests. Am J Respir Crit Care Med 2017;195:120-33.

18 https://rsjh.ca/redcap_v8.5.11.Accessed 4 Sep 2018

19 Baloch R, Jakhrani N, Lal A, et al. Role of dexamethasone for prevention of post-extubation airway obstruction in critically ill adult patients. J Surg Pak 2010;15:3-8.

20 Smith OM, McDonald E, Zytaruk N, et al. Enhancing the informed consent process for critical care research: strategies from a thromboprophylaxis trial. Intensive Crit Care Nurs 2013;29:300-9.

21 Abbade LPF, Abbade JF, Thabane L. Introducing the CONSORT extension to pilot trials: enhancing the design, conduct and reporting of pilot or feasibility trials. J Venom Anim Toxins Incl Trop Dis 2018;2:4.

22 Birkett MA, Day SJ. Internal pilot studies for estimating sample size. Stat Med 1994:13:2455-63.

23 Eldridge SM, Chan CL, Campbell MJ, et al. CONSORT 2010 statement: extension to randomised pilot and feasibility trials. BMJ 2016;355:i5239.

24 Alhazzani W, Guyatt G, Alshahrani M, et al. Withholding pantoprazole for stress ulcer prophylaxis in critically ill patients: a pilot randomized clinical trial and meta-analysis. Crit Care Med 2017;45:1121-9.

25 Medina CR, Camargo JdeJ, Felicetti JC, et al. Laceraçãotraquealpósintubação: análise de trêscasoserevisão de literatura. J Bras Pneumol 2009;35:809-13.

26 Altinok T, Can A. Management of tracheobronchial injuries. Eurasian J Med 2014;46:209-15

27 Law JA, Broemling N, Cooper RM, et al. The difficult airway with recommendations for management--part 1--difficult tracheal intubation encountered in an unconscious/induced patient. Can $J$ Anaesth 2013;60:1089-118. 